\title{
EIDSS Application for CCHF Foci Activity Epi-Analysis and Prediction in Kazakhstan
}

\author{
Stanislav V. Kazakov ${ }^{1}$, Alexey V. Burdakov*2, Kenes S. Ospanov ${ }^{3}$, Aizhan S. \\ Esmagambetova ${ }^{4}$, Andrey O. Ukharov ${ }^{2}$, Veronika P. Sadovskaya ${ }^{1}$ and Umirbek B. Usenov ${ }^{3}$
}

${ }^{1}$ Kazakh Scientific Center of Quarantine and Zoonotic Diseases, Almaty, Kazakhstan; ${ }^{2}$ Black \& Veatch, Overland Park, KS, USA; ${ }^{3}$ Scientific Center of Sanitary and Epidemiological Expertise and Monitoring, Almaty, Kazakhstan; ${ }^{4}$ Committee of State Sanitary and Epidemiological Surveillance of the Ministry of Health of Kazakhstan, Astana, Kazakhstan

\section{Objective}

Test of the Electronic Integrated Disease Surveillance System (EIDSS) for epi-analysis and prediction of situation in CCHF foci in Kazakhstan.

\section{Introduction}

CCHF foci are reported in 3 southern regions of Kazakhstan with population of 1 million. The ixodic ticks in the area are CCHF carriers. Human infections ( 3 to 12 cases per year) occur through tick bites and contact with CCHF patient blood. CCHF epidemiological process in Kazakhstan has prominent seasonality (spring-summer period) and the rhythm of epidemic appearances (5-6-years). The rhythmical population incidence rate is associated with natural and climate factors, which govern the increase in the number of ixodic ticks, their infection rate (virus carrier state), and directly correlates with the population density and the livestock number that are the principal tick feeders in nature.

\section{Methods}

EIDSS version 4 provides capability to collect, share and process epidemiological, clinical and laboratory data on infectious diseases in medicine, veterinary and environment sectors. It is currently deployed in Kazakhstan at 150 sites of the Ministry of Agriculture (planned up to 271), and at 8 sites of the Ministry of Health (planned up to 23).

Three available indicators (for 2007-2011) were used for analysis: population; tick infection rate (relative density of CCHF seropositive tick samples per total number of tested laboratory samples); CCHF human case rate by districts per $10^{\prime} 000$.

The following procedure was conducted:

1) Demographic information, diagnosis and location data entry into EIDSS

2) Tick collection location data, total number of tested samples (pools), and number of seropositive data entry into EIDSS

3) Correlation joint analysis of data on vectors and epidemiological surveillance in Analysis, Visualization and Reporting (AVR) module

\section{Results}

EIDSS generated 12 different maps filtered according to the selected regions, ticks, demographics and CCHF human incidence, aggregated by region, correlated by 3 indicators entered into the database. This allowed visualizing information to support epi-analysis. As a result, for each of the 3 regions specific districts with the highest risk of the CCHF epidemic outbreaks were identified. The resulting information was grouped into 3 clusters of risk with the following criteria: population density, tick infection rate and human cases for each of 25 CCHF-disadvantaged districts (see map). These results predict the epidemic situation in a particular area and support management decisions for planning and correction of preventive antitick and anti-epidemic measures and funding requirements.

\section{Conclusions}

EIDSS with Natural Vectors and the AVR modules has capabilities for analysis and prediction of epizootic and epidemic processes in vector-borne virus infections foci. It is an easy to use and free-ofcharge tool that can become the basic instrument for especially dangerous diseases field epidemiologists as well as for the ministries and local governments for CCHF prophylaxis decision support.

\section{Keywords}

CCHF; one health; electronic disease surveillance; EIDSS; multi-factor analysis

\section{References}

1. Kazakov S.V., et.al. CCHF Causation model in the Roitman's ring graphs // Hygiene, Epidemiology and Immunobiology, 2000, Nos.34, pp.88-90

2. Durumbetov E.E., et.al. Practical Application of Epidemiological Triad Model for Managerial Decision-Making within the System of EpiSurveillance in Moyinkum Foci of CCHF // Proceedings of New Technologies in Medicine and Pharmacy, Astana 2001, pp.34-35

3. Kazakov S.V., et.al. Methodological Approaches to Study Epidemic Process of CCHF in Moyinkum Foci of Zhambyl Region. // Hygiene, Epidemiology and Immunobiology, 2001. Nos.1-2, pp.75-84

4. Onishchenko G.G., et.al. Using IFA and RT-PCR in Study of Ixodic Tick Infection Rate Collected in CCHF Foci in Kazakhstan and Tajikistan in 2001-02. // Virology Matters. - M., 2005. No.1 - pp.23-27

5. Ospanov K.S., Kazakov S.V., et.al. On Prospects of Further Study of CCHF Foci in Kazakhstan // Science and Disease Surveillance Review, BTRP, Atlanta, 2009, p.126

6. Burdakov A., Ukharov A. Transforming national human and veterinary disease surveillance systems from paper into integrated electronic form in the FSU countries // 15th International Congress on Infectious Diseases (ICID), Bangkok 2012

\footnotetext{
*Alexey V. Burdakov

E-mail: BurdakovAV@bv.com
} 